\title{
Low power wake-up receiver based on ultrasound communication for wireless sensor network
}

\author{
Yan Chiew Wong ${ }^{1}$, Szi Hui Tan², Ranjit Singh Sarban Singh ${ }^{3}$, Haoyu Zhang ${ }^{4}$, \\ A. R. Syafeeza ${ }^{5}$, N. A. Hamid \\ ${ }^{1,2,3,5,6}$ Centre for Telecommunication Research \& Innovation (CeTRI), Fakulti Kejuruteraan Elektronik dan Kejuruteraan \\ Komputer (FKEKK), Universiti Teknikal Malay sia Melaka (UTeM), M alay sia \\ ${ }^{4}$ Zhejiang Ocean University, School of Naval Architecture and Mechanical -electrical Engineering, China
}

\begin{tabular}{|c|c|}
\hline Article Info & ABSTRACT \\
\hline Article history: & Wireless sensor network (WSN) consists of base stations and sensors nodes \\
\hline Received Jun 10, 2019 & to monitor physical and environmental conditions. Power consumption \\
\hline Revised Aug 20, 2019 & is required for the main transceiver in WSN to receive communication \\
\hline Accepted Sep 26, 2019 & $\begin{array}{l}\text { requests all the time. Hence, a low power wake-up receiver is needed to } \\
\text { minimize the power consumption of WSN. In this work a low power }\end{array}$ \\
\hline Keywords: & $\begin{array}{l}\text { wake-up receiver using ultrasound data communication is designed. } \\
\text { Wake-up receiver is used to detect wake-up signal to activate a device }\end{array}$ \\
\hline CMOS & in WSN. Functional block modelling of the wake-up receiver is developed \\
\hline RF data communication & $\begin{array}{l}\text { in Silterra CMOS } 130 \mathrm{~nm} \text { process technology. The performance of } \\
\text { the wake-up receiver has been analyzed and achieving low power }\end{array}$ \\
\hline Ultrasound data communication & consumption which is $22.45 \mu \mathrm{W}$. A prototype to demonstrate a wireless \\
\hline Wake-up receiver & sensor node with wake-up receiver has been developed incorporating both \\
\hline Wireless sensornetworks & $\begin{array}{l}\text { ultrasonic and RF for internal and external communication respectively. } \\
\text { We achieve } 99.97 \% \text { of power saving for } 10 \text { s operation in the experimental } \\
\text { setup for the WSN with and without wake-up receiver. Wake-up receiver } \\
\text { used in WSN save power and prolong the lifetime of batteries and thus } \\
\text { extending the operational lifetime of WSN. }\end{array}$ \\
\hline
\end{tabular}

This is an open access article under the CC BY-SA license.

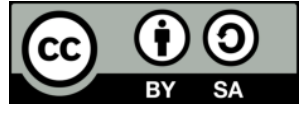

\section{Corresponding Author:}

Yan Chiew Wong,

Micro and Nano Electronic Research Group,Centre for Telecommunication Research and Innovation,

Fakulti Kejuruteraan Elektronik dan Kejuruteraan Komputer,Universiti Teknikal Malaysia Melaka,

Hang Tuah Jaya, 76100 Durian Tunggal, Melaka, Malaysia.

Email: ycwong@utem.edu.my

\section{INTRODUCTION}

A wireless sensor network (WSN) is a wireless network that consists of base stations and a large amount of spatially distributed and dedicated sensors to monitor physical and environmental conditions such as pressure, temperature, motion, sound vibration or pollutants at different locations. WSN consists of many sensor nodes to collect sensory data, to perform some processing and to communicate with other connected nodes in the network [1-2]. WSN provides various uses in many fields such as urgent disaster management, critical military mission, precise agricultural production, intelligent home appliances and so forth [3-4].

Power consumption is a main constraint in WSN as the operational lifetime of WSN is considered as a critical issue for deployment of the network [5-9]. Sensor nodes in WSN are powered by batteries which need long operating hours without sacrificing system performance but the power of the batteries is limited and exhausts after some time [10-12]. Due to hundreds to thousands of distributed nodes in WSN, the replacement of batteries becomes difficult and costly [13-15]. Main transceiver plays an important role 
in WSN as it provides the capability for wireless communication to the sensor nodes but it requires high power consumption for working. Main transceiver operates according to the duty-cycle schedule or checks for communication requests periodically [16]. The main transceiver receives a communication request when it is in active mode and this may cause a failure to receive communication request if it is inactive. When the main transceiver is turned on, the sensor node operates to transmit or receive data. If there is data to be transmitted but no data to be received by the sensor node, the power is still consumed for listening [17]. Thus, energy consumption for wireless communication in WSN usually dominates overall power consumption shown in Figures 1(a) and (b).

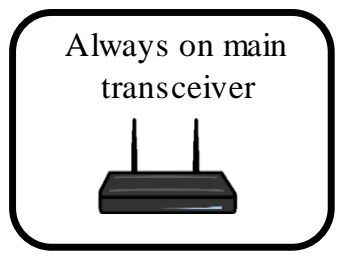

(a)

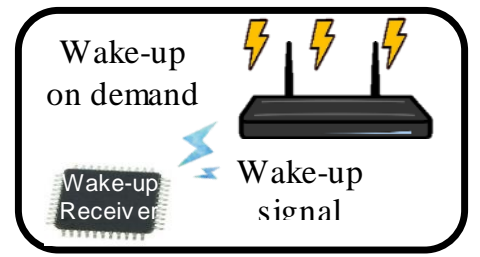

(b)

Figure 1. WSN, (a) Without wake-up receiver and,(b) With wake-up receiver

In order to minimize the power consumption in WSN, a wake-up receiver with low power consumption is needed. A wake-up receiver always active and capable to receive communication requests all the time. The wake-up receiver able to monitor the channel continuously, listen for a wake-up signal from other nodes and activate the main transceiver upon detection. Once the wake-up receiver receives a wake-up request from the nodes, it sends the wake-up signal to the main transceiver for activation purpose. The main transceiver is in active state only in an on-demand way by using a wake-up receiver in an energy efficient network operation.

Based on [18], the wake-up receiver with low power consumption had been achieved by using radio frequency (RF) communication system but it required higher carrier frequency to process compared to other communication system. Wake-up receiver using RF communication system consumed higher power than other communication system. In [19], the wake-up receiver designed consumed $51 \mu \mathrm{W}$. Optical communication system had been used for designing a low power wake-up receiver [20] but the performance of visible light communication system will be affected by the interference from other light sources. According to [21], a wake-up receiver designed by using infrared communication system achieved low power consumption but the communication system has its weakness which it requires accurate alignment of a narrow beam in order to get a better communication. In this paper, a low power wake-up receiver is proposed by using ultrasound data communication [22-25]. The carrier frequency is set at $40 \mathrm{kHz}$ which is lower than RF communication system. This paper is organized in four section. In section 2, the methodology of this work is explained for functional block modelling and prototyping. Section 3 presents the functional block modelling of the wake-up receiver whereas section 4 demonstrates a wireless sensor node with wake-up receiver using a prototype.

\section{RESEARCH METHODOLOGY}

A functional block modelling of low power wake-up receiver consists of low noise amplifier, carrier band amplifier and mixer design as shown in Figure 2. The circuit is designed using Silterra 130nm CMOS process technology. For the LNA design, S-parameter analysis is performed. $S_{11}$ and $S_{22}$ must less than -10dB while $S_{12}$ must less than $S_{21}$ and the value must less than $-12 \mathrm{~dB}$. $S_{22}$ is also known as power gain. The value of $S_{22}$ must greater than 0 . The phase margin of the carrier band amplifier is measured to determine the stability of the amplifier. The acceptable range of phase margin is greater than $45^{\circ}$ but less than $180^{\circ}$. A periodic steady state (PSS) analysis and periodic XF (PXF) analysis are performed to obtain conversion gain of mixer. A prototype is built to demonstrate a sensor node with wake-up receiver using ultrasound data communication. 


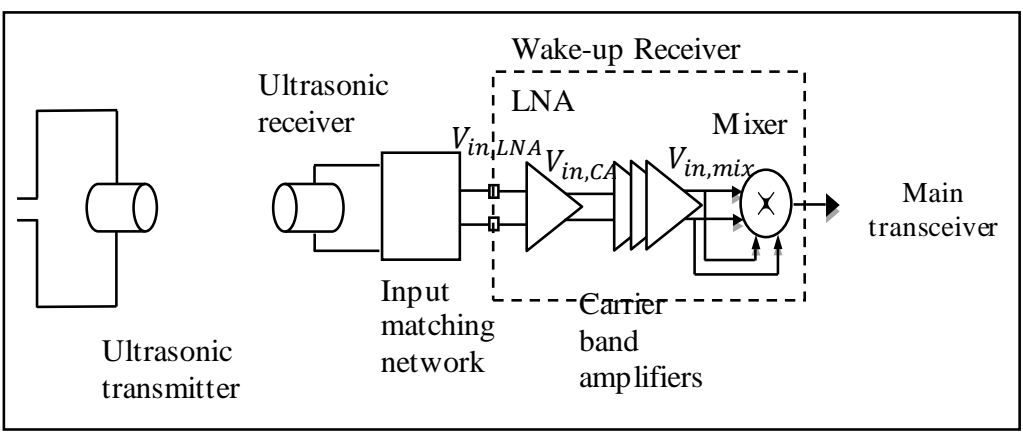

Figure 2. Modelling of low power wake-up receiver for WSN

\section{FUNCTIONAL BLOCKS IN WAKE-UP RECEIVER}

The functional block modelling of wake-up receiver using ultrasound data communication is designed for low noise amplifier, carrier band amplifier and mixer by using Cadence Virtuoso. Simulations are executed according to the design specifications of the circuits as presented in Section 2.

\subsection{Low noise amplifier}

Low noise amplifier (LNA) can be used to replace amplifiers with higher internal noise levels in order to enhance the signal-to-noise ratio of the RF receiver system. External devices and internal circuit components contribute to the noise signals in the system. The noise signal must be reduced to avoid degradation of signals and to maximize the signal-to-noise ratio of the system.

$$
\begin{aligned}
& \text { Gain }=-G_{m} R_{L} \\
& N F=\frac{\text { Total noise power at output }}{\text { noise power at output due to input source }}
\end{aligned}
$$

The schematic of the LNA consists of two-port network, decoupling capacitors, resistors, inductors, and NMOS transistors as shown in Figure 3. Simulation of S-parameter analysis is carried out and the results are shown in Figure 4 and summarized in Table 1.

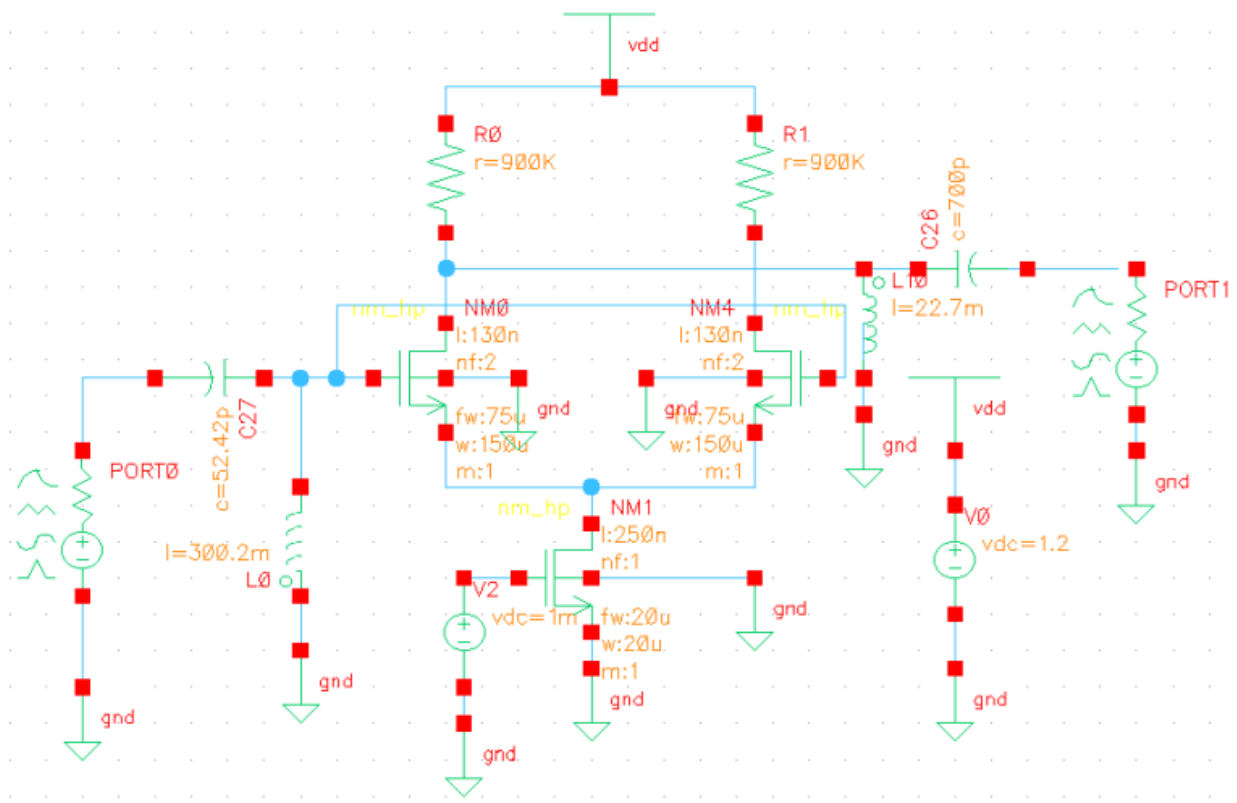

Figure 3. Schematic of low noise amplifier 


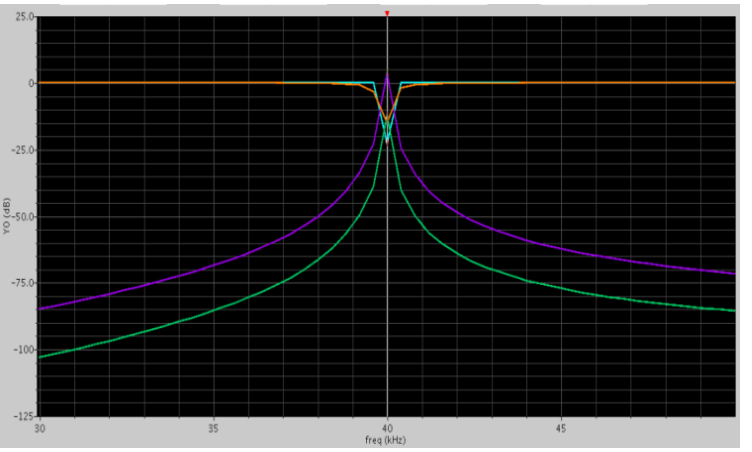

(a)

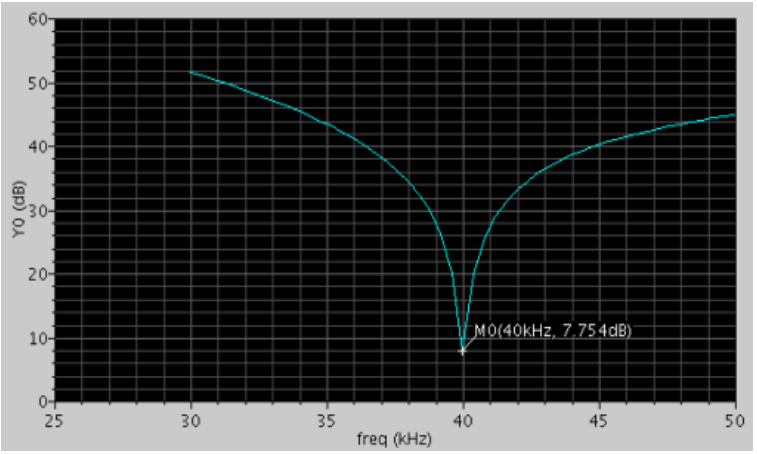

(b)

Figure 4. Simulation result of LNA, (a) $S_{11}, S_{12}, S_{21}$ and $S_{22}$ performance and, (b) Noise figure

Table 1. Design parameter of LNA

\begin{tabular}{ccc}
\hline Design Parameter & Design Specifications & Results $(\mathrm{dB})$ \\
\hline $\boldsymbol{S}_{\mathbf{1 1}}$ & $S_{11}<-10 \mathrm{~dB}$ & -22.44 \\
$\boldsymbol{S}_{\mathbf{1 2}}$ & $S_{12}<-12 \mathrm{~dB}$ & -12.31 \\
$\boldsymbol{S}_{\mathbf{2 1}}$ & $S_{21}>0 \mathrm{~dB}$ & 3.636 \\
$\boldsymbol{S}_{\mathbf{2 2}}$ & $S_{22}<-10 \mathrm{~dB}$ & -14.81 \\
Power gain & Power gain $>0 \mathrm{~dB}$ & 3.636 \\
\multicolumn{2}{c}{ Noise Figure } & 7.754 \\
\hline
\end{tabular}

\subsection{Carrier band amplifier}

A carrier band amplifier is designed to amplify the resulting signal from LNA. NMOS differential amplifier with PMOS load is used in this amplifier. This amplifier consists of common mode feedback, amplifier and buffer as shown in Figure 5. Common mode feedback circuit is a circuit used to sense the common mode voltage, compare the common mode voltage with a proper reference voltage and feedback the correcting common mode signal in order to control the common mode voltage at different nodes that cannot be stabilized by the negative differential feedback. Buffer amplifier is used for providing the transformation of electrical impedance from one circuit to another to prevent the subsequent circuit from loading. The output from LNA is connected with the amplifier and the output is produced in buffer part of the carrier band amplifier. The carrier band signal is amplified from $235.75988 \mathrm{mV}$ to $404.71478 \mathrm{mV}$ as shown in Figure 6.

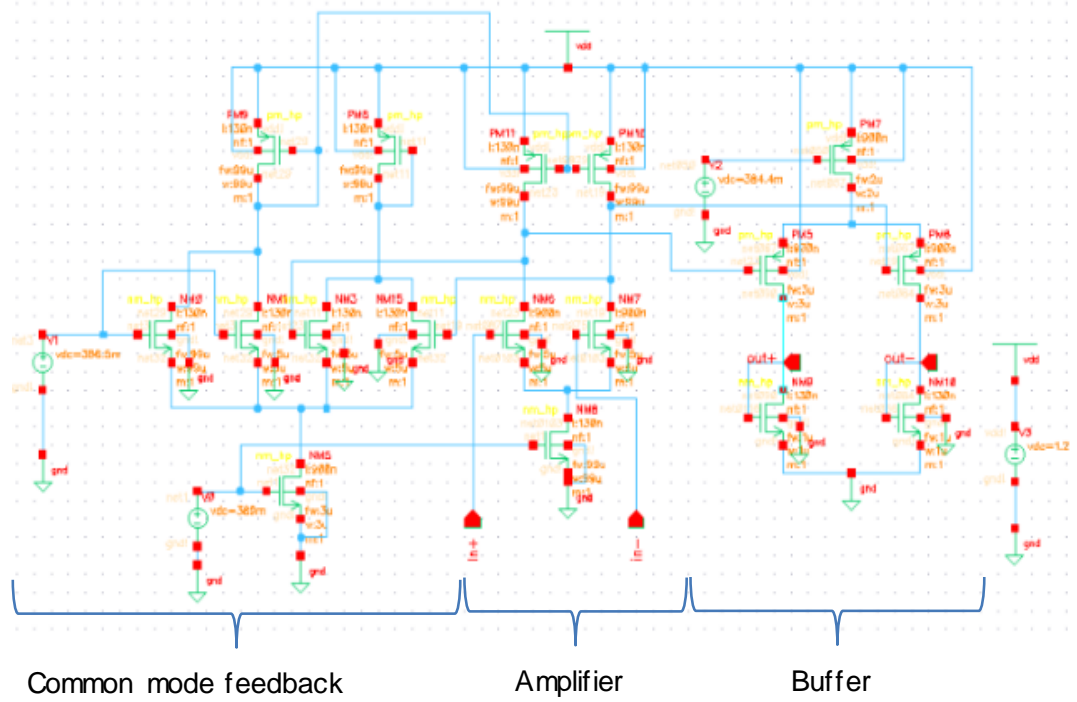

Figure 5. Schematic of carrier band amplifier 


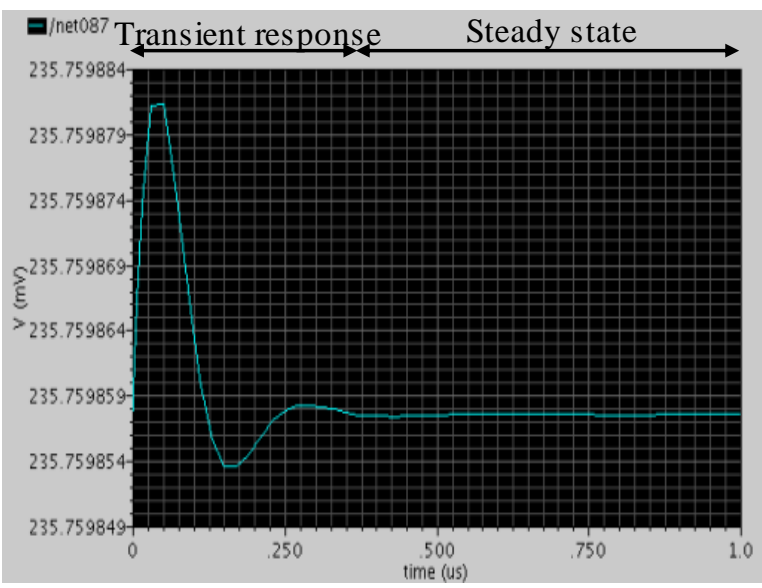

(a)

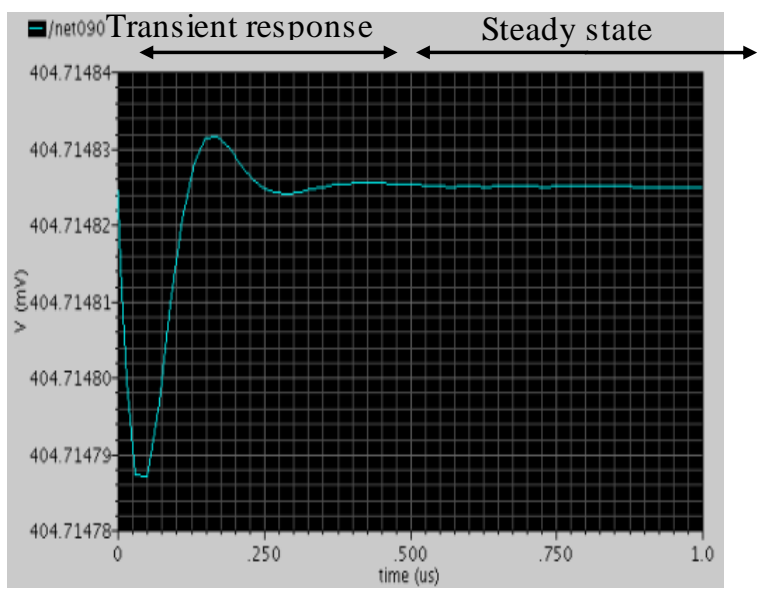

(b)

Figure 6. Transient analys is of carrier band amplifier, (a) Input signal and, (b) Output signal

Stability of the carrier band amplifier has been monitored. The phase margin of the amplifier is $83.75^{\circ}$ as shown in Figure 7 which is in the acceptable range.

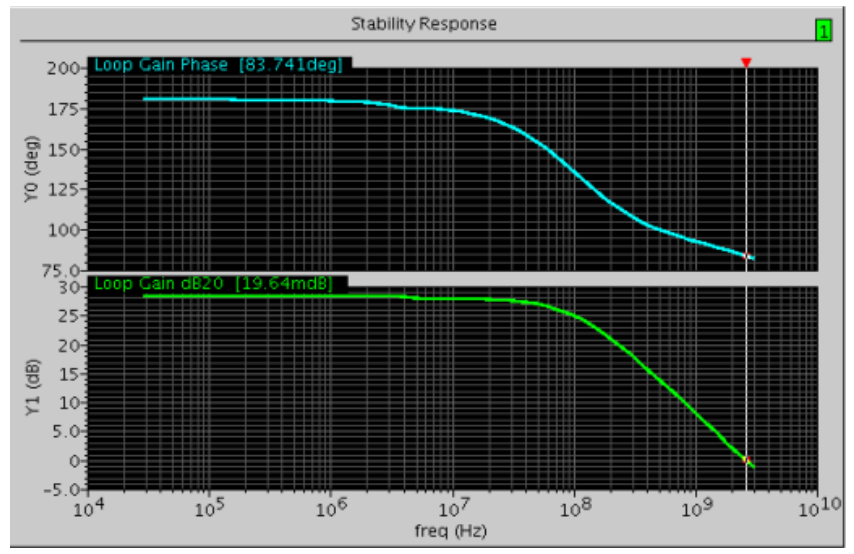

Figure 7. Magnitude and phase of loop gain in decibel units

\subsection{Mixer}

A mixer is a device with three ports that creates new frequency from two signals applied to it. It can be used to modulate or demodulate a signal. The sum of the two input frequencies will be the modulation of signal whereas the difference of the two input frequencies is either modulation or demodulation of the signal as shown in (3). The resulting signal from the carrier band amplifier is down-converted by using a double balanced direct conversion mixer. The mixer consists of LO input port, RF input port, ideal baluns, resistor and NMOS transistors as shown in Figure 8.

$$
f_{I F}=f_{L O} \pm f_{R F}
$$

where $f_{I F}$ is the intermediate frequency which is the output frequency from the sum and difference of two input frequencies, $f_{L O}$ is the local oscillator frequency which is one of the input frequencies and $f_{R F}$ is the input radio frequency. 


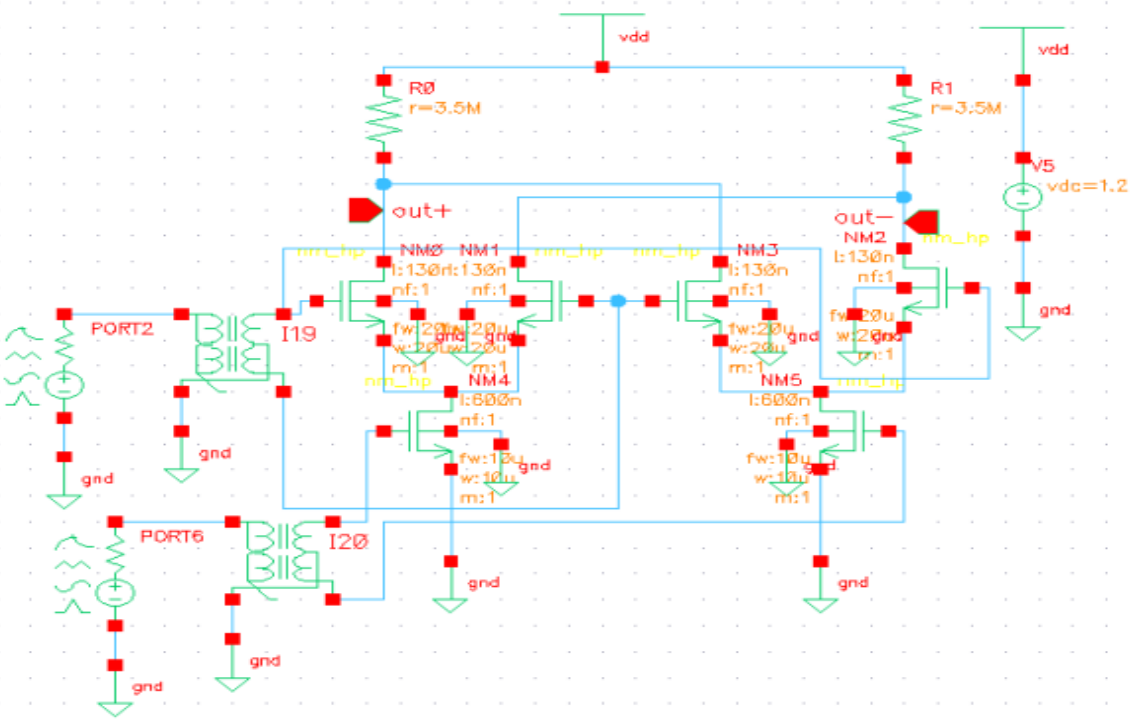

Figure 8. Schematic of mixer

The resulting AC signal from the carrier band amplifier is used as RF input port. The output of the mixer is zero-IF as it is a direct conversion mixer that demodulates signal with identical or very close to RF frequency at LO frequency. A periodic steady state analysis (PSS) and periodic XF (PXF) analysis are executed to measure the voltage conversion gain of the mixer. A curve of conversion gain in dB versus IF output frequency is plotted and maximum conversion gain is about $8.8 \mathrm{~dB}$ as shown in Figure 9(a). Noise figure of the mixer is obtained by performing periodic noise analysis as shown in Figure 9(b). The noise figure is reduced with the increasing IF output frequency.

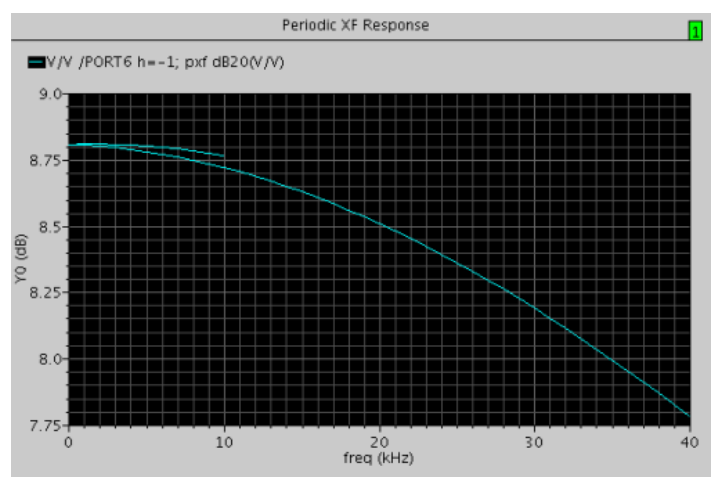

(a)

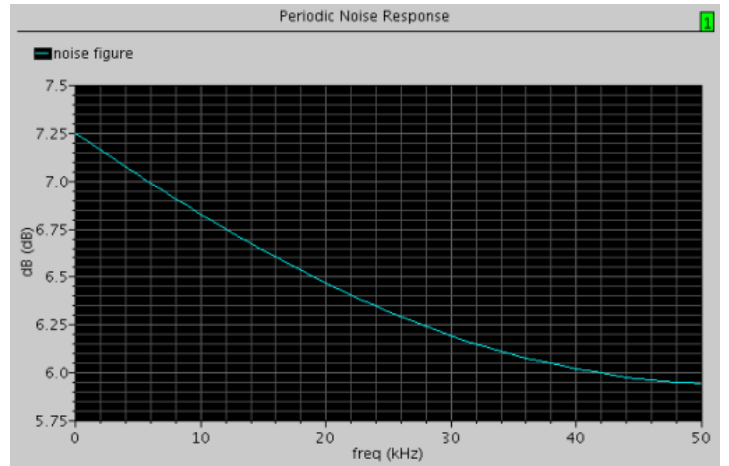

(b)

Figure 9. Double balance mixer, (a) Voltage conversion gain, (b) Noise figure

\subsection{Power consumption of functional block modelling}

The current consumption for LNA, carrier band amplifier and mixer are measured. The current and power consumption of these three schematics are summarized in Table 2.

\begin{tabular}{lcc}
\multicolumn{2}{c}{ Table 2. Current and power consumption of functional block modelling } \\
\hline Functional Block Modelling & Current Consumption & Power consumption \\
\hline LNA & $1.295 \mu \mathrm{A}$ & $1.55 \mu \mathrm{W}$ \\
Carrier Band Amplifier & $17.04 \mu \mathrm{A}$ & $20.45 \mu \mathrm{W}$ \\
Mixer & $376 \mathrm{nA}$ & $0.45 \mu \mathrm{W}$ \\
Total Power Consumption: & & $22.45 \mu \mathrm{W}$ \\
\hline
\end{tabular}




\section{PROTOTYPING}

A prototype is built to demonstrate a sensor node with wake-up receiver using ultrasound data communication. It shows that ultrasound data communication used in internal WSN communication is able to send wake-up signals to activate the RF transmitter which is in sleep mode. The wake-up RF transmitter will transfer data to RF receiver at external devices such as mobile phone, base station, server etc. The WSN which consists of wake-up receiver based on ultrasonic communication system and RF transmitter as internal communication is shown in Figure 10(a) whereas external communication through RF receiver is shown in Figure 10(b).

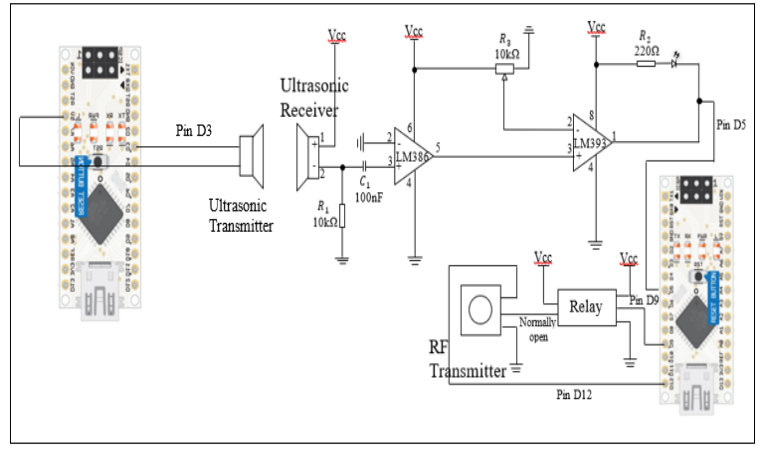

(a)

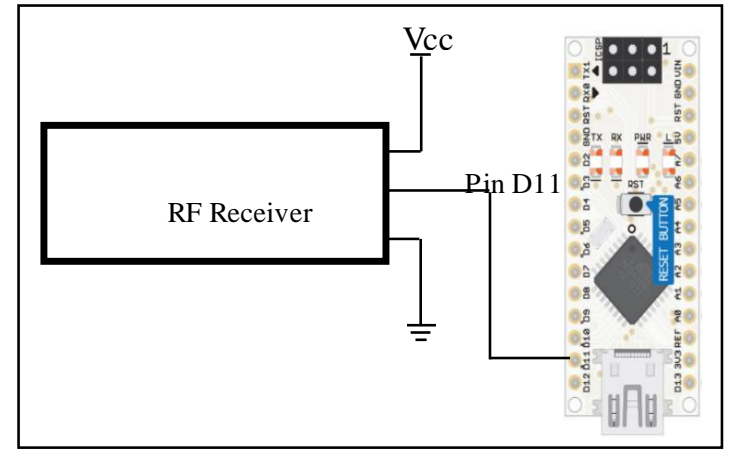

(b)

Figure 10. Circuit diagram of wake up receiver for WSN, (a) Internal communication through ultrasound,

(b) External communication through RF

When there is no demand, a relay is used to turn RF transmitter into sleep mode. To wake up the RF transmitter, an ultrasonic transmitter sends wake-up signal as tone to the ultrasonic receiver to acknowledge the receiver to wake RF transmitter. When a wake-up signal is received, the interrupt pin and the relay are in active low state. The relay wakes the RF transmitter up by supplying voltage to the transmitter to operate and the interrupt pin activates the RF receiver. A string of "Hello World" is set in RF transmitter to send to the RF receiver when they are activated. The string is received by the RF receiver. When there is no tone received by the ultrasonic receiver, the relay and interrupt pin of the Arduino are in active high which deactivate the RF transmitter and receiver. RF transmitter and receiver are in sleep mode again. No data can be transmitted or received by the RF communication system.

The power consumption of the prototype is measured by using a USB power monitor. The power consumption is measured at the power supply of the RF receiver as shown in Table 3. The power consumption of the RF receiver to receive the data transmitted from RF transmitter in one second is $0.065 \mathrm{~W}$ whereas the power consumption of the RF receiver in sleep mode in one second is $0.031 \mathrm{~W}$. The RF receiver receives two data in 10 seconds. To find the total power consumption for 10 seconds, the power consumption is measured by using the formula of (4).

$$
\text { Energy }=P_{\text {standby }} * t+P_{\text {receive } d_{\text {dat }}} * t
$$

where: $P_{\text {total }}=$ total power consumption of the RF receiver

$P_{\text {standby }}=$ power consumption of the RF receiver in sleep mode

$P_{\text {received_data }}=$ power consumption of the $\mathrm{RF}$ receiver in active mode.

The power consumption of the RF receiver without wake-up receiver in the sensor node is measured. The RF receiver is always active and consumed same power during receiving data or in standby. The power consumption in one second is $0.112 \mathrm{~W}$. A comparis on is made between the power consumption of $\mathrm{RF}$ receiver at external device with and without wake-up receiver of ultrasonic communication system in the sensor node for 10 seconds as shown in Table 3. 
Table 3. Comparison of power consumption between RF receiver with and without wake-up receiver in sensor node

\begin{tabular}{cccc}
\hline Sensor node & $\begin{array}{c}\boldsymbol{P}_{\text {standby }} \text { in 1 } \\
\text { second (W) }\end{array}$ & $\begin{array}{c}\boldsymbol{P}_{\text {received }} \text { ina } \\
\text { second }(\mathrm{W})\end{array}$ & $\begin{array}{c}\text { Energy consumption for 10 seconds } \\
(\text { Wh })\end{array}$ \\
\hline With wake-up receiver & 0.031 & 0.065 & $0.105 \mathrm{~m}$ \\
Without wake-up receiver & 0.112 & 0.112 & 0.311 \\
\hline
\end{tabular}

The power consumption of RF receiver without wake-up receiver in the sensor node is much higher than the RF receiver with wake-up receiver in the sensor node. The power saving is around $99.97 \%$. The developed low power wireless data communication significantly reduces the power consumption and extend the lifetime of the batteries in WSN.

\section{CONCLUSION}

A low power wake-up receiver using ultrasound data communication is designed for wireless sensor node. The functional block modelling of the wake-up receiver is developed in Silterra CMOS 130nm process technology. LNA achieved $S_{11}$ of $-22.44 \mathrm{~dB}, S_{12}$ of $-12.31 \mathrm{~dB}, S_{21}$ of $3.636 \mathrm{~dB}$ and $S_{22}$ of $-14.81 \mathrm{~dB}$ for a good performance of LNA. For the carrier band amplifier, it achieved the amplification of signal and stability of the amplifier. The mixer used to demodulate the resulting signal achieved $8.8 \mathrm{~dB}$ gain and $7.25 \mathrm{~dB}$ noise figure. The total power consumption of the functional block modelling is $22.45 \mu \mathrm{W}$. In order to verify the functionality of ultrasound communication system, a prototype of a wireless sensor node with wake-up receiver is developed. A comparis on of the prototype with and without wake-up receiver in term of the power consumption is performed. More than $99 \%$ of power saving has been achieved. The sensor node with wake-up receiver saves power and extends the lifetime of batteries and the operational lifetime of WSN.

\section{ACKNOWLEDGEMENTS}

The authors acknowledge the technical and financial support by Universiti Teknikal Malaysia Melaka (UTeM).

\section{REFERENCES}

[1] A. Tamizharasi, J. J. Selvathai, A. Kavi Priya, R. Maarlin, and M. Harinetha, "Energy aware heuristic approach for cluster head selection in wireless sensor networks," Bulletin of Electrical Engineering and Informatics, vol. 6, no. 1, pp. 70-75, 2017.

[2] Z. Zhang, A. Mehmood, L. Shu, Z. Huo, Y. Zhang and M. Mukherjee, "A survey on fault diagnosis in wireless sensor networks," in IEEE Access, vol. 6, pp. 11349-11364, 2018.

[3] Shengjun Su and S. Wang, "A simple monitoring network system of wireless sensor network," Bulletin of Electrical Engineering and Informatics, vol. 1, no. 4, pp. 251-254, 2012.

[4] R. Piy are, A. L. Murphy, C. Kiraly, P. Tosato and D. Brunelli, "Ultra low power wake-up radios: a hardware and networking survey," in IEEE Communications Surveys \& Tutorials, vol. 19, no. 4, pp. 2117-2157, Fourthquarter 2017.

[5] F. Liang, L. Zhang, and P. Sun, "Study on the rough-set-based clustering algorithm for sensor networks," Bulletin of Electrical Engineering and Informatics, vol. 3, no. 2, pp. 77-90, 2014.

[6] M. A. R. E. Alselwi, Y. C. Wong, Z. A. F. M. Napiah, "Integrated CMOS rectifier for RF-powered wireless sensor network nodes," Bulletin of Electrical Engineering and Informatics, Vol. 8, No. 3, pp.1-10, 2019.

[7] A. N. F. Asli, Y. C. Wong, “-31 dBm sensitivity high efficiency rectifier for energy scavenging, International Journal of Electronics and Communications," Vol. 91, pp. 44-54, 2018.

[8] R. K. Saini, Ritika, and S. Vijay, "Data flow in wireless sensor network protocol stack by using bellman-ford routing algorithm," Bulletin of Electrical Engineering and Informatics, vol. 6, no. 1, pp. 81-87, 2017.

[9] A. A. Masius, Y. C. Wong, "Miniature high gain slot-fed rectangular dielectric resonator antenna for IoT RF energy harvesting," International Journal of Electronics and Communications, vol.85, pp.39-46, 2018.

[10] I. Jang, D. Pyeon, S. Kim, and H. Yoon, "A survey on communication protocols for wireless sensor networks," J. Comput. Sci. Eng., vol. 7, no. 4, pp. 231-241, 2013.

[11] S. S. S. Ranjit, A. F. M. F. Aqil, S. S. S. Baljit, Y. C. Wong, "Review of communication methods and system design structure for solar monitoring system," AIP Conference Proceedings, 2018.

[12] U. K. Singh, K. C. Phuleriya, K. Bunkar, and S. Bhumarkar, "Exploration of wireless sensor networks technology and development," Int. J. Emerg. Trends Technol. Comput. Sci., vol. 1, no. 1, pp. 69-72, 2012.

[13] M. Prinn, L. Moore, M. Hayes, and B. O. Flynn, "Comparing low power listening Techniques with Wake-up receiver technology," Third Int. Conf. Smart Syst. Devices Technol. (SMART 2014), pp. 88-93, 2014. 
[14] Y. C. Wong, C. W. Tan, S. S. S. Ranjit, A. R. Syafeeza and N. A. Hamid, "Energy scavenging for mobile and wireless devices using CMOS rectifier circuit," 2019 IEEE International Conference on Industrial Technology (ICIT), Melbourne, Australia, 2019, pp. 429-433.

[15] A. M. Alphonsos, Y. C. Wong, "Design of high gain co-planar waveguide (CPW) fed staircase shaped monopole antenna with modified ground plane for radio-frequency (RF) energy harvesting application," Mechanical Engineering Research Day 2017, pp. 118-119, 2017.

[16] S. Bdiri, F. Derbel, and O. Kanoun, "A tuned-RF duty-cycled Wake-up receiver with -90 dBm sensitivity," Sensors, vol. 18, no. 1, pp. 1-21, 2018.

[17] N. M. Pletcher, "Ultra-low power Wake-up receivers for wireless sensor networks," Thesis, 2008.

[18] K. Cheng and S. Chen, "An ultralow-power Wake-Up receiver based on direct active RF detection," in IEEE Transactions on Circuits and Systems I: Regular Papers, vol. 64, no. 7, pp. 1661-1672, July 2017.

[19] X. Huang, S. Rampu, X. Wang, G. Dolmans and H. de Groot, "A 2.4GHz/915MHz 51 $\mu$ W wake-up receiver with offset and noise suppression," 2010 IEEE International Solid-State Circuits Conference - (ISSCC), San Francisco, CA, 2010, pp. 222-223.

[20] G. Kim et al., "A 695 pW standby power optical Wake-up receiver for wireless sensor nodes," Proc. Cust. Integr. Circuits Conf., pp. 1-4, 2012.

[21] H. Ishihara et al., "A $130 \mu \mathrm{A}$ wake-up receiver SoC in $0.13 \mu \mathrm{m}$ CMOS for reducing standby power of an electric appliance controlled by an infrared remote controller," 2011 IEEE International Solid-State Circuits Conference, San Francisco, CA, 2011, pp. 226-228.

[22] K. Yadav, I. Ky missis and P. R. Kinget, "A 4.4-\$\muW Wake-up receiver using ultrasound data," in IEEE Journal of Solid-State Circuits, vol. 48, no. 3, pp. 649-660, March 2013.

[23] H. Fuketa, S. O'uchi and T. Matsukawa, "A 0.3-V 1-uW super-regenerative ultrasound Wake-up receiver with power scalability," in IEEE Transactions on Circuits and Systems II: Express Briefs, vol. 64, no. 9, pp. 1027-1031, Sept. 2017.

[24] W. Jiang and W. M. D. Wright, "Multichannel ultrasonic data communications in air using range-dependent modulation schemes," in IEEE Transactions on Ultrasonics, Ferroelectrics, and Frequency Control, vol. 63, no. 1, pp. 147-155, Jan. 2016.

[25] A. S. Rekhi and A. Arbabian, "A $14.5 \mathrm{~mm} 28 \mathrm{nW}-59.7 \mathrm{dBm}$-sensitivity ultrasonic wake-up receiver for power-, area-, and interference-constrained applications," 2018 IEEE International Solid-State Circuits Conference-(ISSCC), San Francisco, CA, 2018, pp.454-456. 